Portland State University

PDXScholar

\title{
A Solvable Model for Gravity Driven Granular Dynamics
}

\author{
J. J. P. Veerman \\ Portland State University, veerman@pdx.edu
}

Follow this and additional works at: https://pdxscholar.library.pdx.edu/mth_fac

Part of the Non-linear Dynamics Commons

Let us know how access to this document benefits you.

\section{Citation Details}

Veerman, J. J. P., "A Solvable Model for Gravity Driven Granular Dynamics" (2005). Mathematics and Statistics Faculty Publications and Presentations. 137.

https://pdxscholar.library.pdx.edu/mth_fac/137

This Post-Print is brought to you for free and open access. It has been accepted for inclusion in Mathematics and Statistics Faculty Publications and Presentations by an authorized administrator of PDXScholar. Please contact us if we can make this document more accessible: pdxscholar@pdx.edu. 


\title{
A Solvable Model for Gravity Driven Granular Dynamics
}

\author{
J. J. P. Veerman * \\ Department of Mathematics and Statistics, \\ Portland State University, Portland, OR 97207.
}

February 19, 2005

\begin{abstract}
We discuss a toy model to study the dynamics of individual particles in avalanches. The model describes a particle launched from an inclined infinite staircase. The particle is not allowed to bounce when it collides with the staircase. During the collision, the particle loses some energy, and after that slides on to the end of the step it landed on. The process then repeats itself.

The dynamics of this no-bounce model can essentially be completely understood. Partial versions of some results were stated and argued in previous work $[16,17]$. Here we give a full description together with all the proofs. We also briefly mention a second model where the no-bounce restriction is lifted and briefly describe the main features of that more complicated model (see $[18,19])$.
\end{abstract}

KEYWORDS: Dynamical Systems, Granular Flow, Periodic Orbits, Instability.

\section{Introduction}

The purpose of this note is to give a mathematical treatment of the dynamics of a particle falling down an inclined slope with a simple periodic profile (a "staircase"). The idea is to develop new insights about the dynamics of individual particles in an "avalanche" motion. Such motions happen in granular material when it is poured on a pile. This is a currently very active area of research with many applications (see $[8,9]$ ). Our basic question is: What causes an avalanche, and what are the circumstances under which it stops?

As an example, think of dropping sand on top of a sand pile. Initially the pile will become steeper and steeper. But finally, the slope will stop increasing and the falling particles will tend to roll far down the mound. The individual particle rolling down this pile "sees" a very rugged irregular landscape which we have modelled as a staircase. Whenever the particle collides with the staircase it loses some of its energy.

The equations for any realistic model of such a process would be much too complicated to glean substantial analytic insight from. What we therefore try to develop is a paradigm for this kind of dynamics. The model in this note has various interesting features that enable us to treat it analytically.

While most other models have concentrated on the collective aspects of avalanches - that is: the interaction of many particles with one another (for example by considering the granular particles as a fluid [11], or a cellular automaton [1]. See [9] for an overview) - we are more interested in the individual motion of particles. Thus the first simplifying feature is that we have opted for a dynamical system describing the motion of a single particle. This approach is also followed by [13] and references therein. To eliminate another substantial complication, namely the detailed description of the motion of a rolling particle on a surface (see [6]), we assume that our particle is a point particle (similar to [15]). Lastly, the nature of the process by which a colliding particle loses some its energy is not very well understood (see [3], [2], [14]). In

*e-mail: veerman@pdx.edu 
fact, even the "law of restitution" - the rule that gives the velocity "just" after the collision as a function of the velocity "just" before the collision - is not very well understood (same references). In this note we avoid this problem by showing that the obtained results do not depend on the details of the law of restitution.

We now make precise the aims of our analysis. It has been shown in experiments (see [7]) that the slope of a pile of (dry) granular material just after an avalanche, is always the same (though it may depend on the type of material). This angle, $\theta_{r}$, is called the angle of repose. If the slope of the pile slowly increased then a new avalanche starts at $\theta_{m}$, the maximal angle of stability. Typically the difference between $\theta_{m}$ and $\theta_{r}$ is a few degrees. We present two - for mathematical purposes - highly idealized versions of this physical problem. The purpose is to investigate what kinds of dynamical models can lead to a qualitatively similar behavior characterized by 3 regimes consistent with the experiments. In particular our second model (in section 5) shows that this peculiar behavior can be exhibited by a single particle model.

We now make precise the aims of our analysis which were stated more informally in [18] and [19]. Consider a point particle falling down an infinite staircase (the details of the model do not matter here). Let $X$ be the "dynamical" space (position and velocity) and $P$ the space of "parameters" (such as the angle by which the staircase is tilted and the coefficient of restitution). Now suppose that the particle starts at $t=0$ with initial conditions $x_{0} \in X$ and the parameters set to $p_{0} \in P$. We can define the criterion map

$$
\sigma: X \times P \rightarrow\{I, I I, I I I\}
$$

where a point in $\left(x_{0}, p_{0}\right) \in X \times P$ is assigned a $I$ if the orbit with initial condition $x_{0}$ and with the parameters fixed and equal to $p_{0}$ will eventually be attracted to a fixed point where the velocities are all equal to zero (the particle stops). Similarly, a point $\left(x_{1}, p_{1}\right) \in X \times P$ is assigned a $I I I$ if the orbit with initial condition $x_{1}$ and with the parameters fixed and equal to $p_{1}$ has the property that the velocity as a function of time is unbounded. We assign the value $I I$ if neither is the case. Notice that in this category belong the kinds orbits usually studied in dynamical systems, stable periodic or unstable periodic orbits, strange attractors, and so forth. For any given class of models, we will try to understand, first, the partition that $\sigma$ induces on $X \times P$, and, as a secondary aim, we try to partition $\sigma^{-1}(I I)$ further according to qualitative characteristics such as existence and stability of certain orbits.

We should emphasize that this division of phase-space is akin but not identical to the dynamical regimes $\mathrm{A}, \mathrm{B}$, and $\mathrm{C}$, set forth in [13]. In particular the boundary between $\mathrm{B}$ and $\mathrm{C}$ (see also [5])and that between $\mathrm{B}$ and $\mathrm{C}$ (for example in [12]) are concepts similar to the boundary between $I$ and $I I$ on the one hand and $I I$ and $I I I$ on the other. The problem is to formulate models that are mathematically tractable but still show this behavior. An elegant model but insufficiently rich dynamics to show this behavior was developed in [15]. Our first model shows surprisingly rich dynamics but can essentially be completely understood. Unfortunately, it shows only two of the three regimes. We offset this analysis with a more sophisticated model which is harder to analyze but appears to have three clear dynamical regimes.

In section 2 we will start by giving the physical description of the model and derive the resulting dynamical system. The model describes a particle launched from an inclined infinite staircase. The particle is not allowed to bounce up when it collides with the staircase. During the collision, the particle loses some energy, and after that slides on to the end of the step it landed on. The process then repeats itself. We remark here that numerical runs of this very simple model, in some cases mimic actual physical experiments remarkably well as was argued in [16].

In sections 3 and 4 we will see that, somewhat surprisingly, the dynamics of this model can be essentially completely understood and that this understanding is (up to a point) independent of the details of the assumed law of restitution. We note in passing that the dynamics of this model also exhibits a surprising similarity to physical experiments done with falling particles (for this comparison see [16]). Partial versions of some results in this article were stated and argued in previous work (see [16] and [17]).

Finally, in section 5, we will mention a somewhat more sophisticated class of models model and summarize the main results. This discussion is based on [18] and [19]. The fact that the slope of a dry sand pile upon which sand is continually poured will typically lie in a certain interval, finds, again somewhat surprisingly, theoretical support in this model. (A hybrid model is also discussed in [19].) 


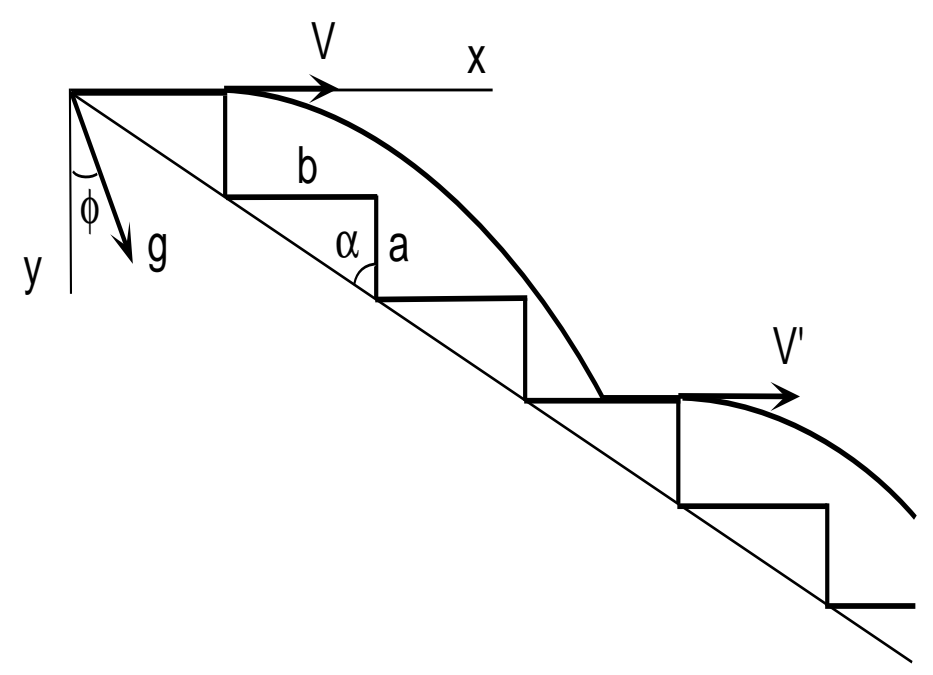

Figure 2.1: Dynamics of the no-bounce model.

Acknowledgements: I would like to thank Giovani Vasconcelos for many useful suggestions.

\section{The Model}

We now describe the model. Our description is very similar to the one in [17]. The situation is depicted in Figure 2.1. A particle of mass $m>0$ is falling down a simple staircase shape whose steps have height $a$ and length $b$. For convenience, we choose a system of coordinates in such a way that the step plateaus are aligned with the $x$ axis and the direction of the acceleration of gravity $\mathrm{g}$ makes an angle $\phi$ with the $y$ axis. The staircase is given a forward inclination of angle $\phi$. To get a consistent model we require $\tan \phi \in[0, b / a]$.

The particle is launched at the top of the "staircase" with a given initial velocity $\left(v_{x}, 0\right)$ where $v_{x}>0$. The particle will undergo a ballistic flight until it collides with another plateau located a certain number $n \in \mathbb{N}$ of steps below the departure step (e.g., $n=3$ in the figure). Accordingly, we will refer to the integer $n$ as the jump number associated with this flight. A point particle in free flight undergoes a uniform downward gravitational acceleration which we denote by $g$. Then if the jump number is $n$, the time of flight to the next landing is $T$, the velocity just before that landing $(u, v)$ and the displacement are $(x, y)$ determined by:

$$
\begin{aligned}
& u=g s T+v_{x} \\
& v=-g c T \\
& x=\frac{1}{2} g s T^{2}+v_{x} T \\
& y=-\frac{1}{2} g c T^{2}=-n a
\end{aligned}
$$

(Here $s \equiv \sin \phi$ and $c \equiv \cos \phi$ ). From the last of these equations, we can of course, solve for $T$ and thus find the coordinates of the particle just prior to the collision.

It is easy to see, under our hypotheses, that the dynamics would be trivial if energy is conserved: all initial conditions would lead to orbits with unbounded velocity (category $I I I$ in the introduction). It is much more realistic to assume that the particle loses some of its energy when it hits the step upon which it falls. We will assume that the $y$-component of the velocity of the particle immediately after the collision is zero (the "no vertical bounce" condition), and its $x$-component $v_{x}^{\prime}$ is given by

$$
u^{\prime}=e C(u, v)
$$

Here, $e \in[0,1]$ is a fixed parameter, and $C: \mathbb{R}^{2} \rightarrow[0, \infty)$ is a function satisfying: 
$C$ is continuously differentiable and homogeneous of degree 1, that is: $C(\alpha u, \alpha v)=\alpha C(u, v)$.

$$
C(0,-1)=0 \text {. }
$$

For all $u>0: \partial_{u} C(u,-1) \in[0,1]$.

Lemma 2.1 Note that the above implies that for all fixed $\alpha<0$ :

$C(0, \alpha)=0, \partial_{u} C(u, \alpha) \in[0,1]$, and thus $0 \leq C(u, \alpha) \leq u$.

The customary way to write a restitution law is: $u^{\prime}=\mu(u / v) \cdot u$ where $u / v$ is the tangent of the angle between the velocity vector and the perpendicular to the surface. Suppose that $v$ is bounded away from zero and the coefficient of restitution $\mu$ is smooth with $u \cdot \mu^{\prime}(u)+\mu(u) \in[0,1]$. It is important to note that restitution laws of this type satisfy the requirements given above.

Finally, we will assume that after the collision the particle slides to the end of the ramp without friction. This means that throughout this part of the motion, energy is conserved. Since the particle slides a distance $n b-x$ on a ramp that makes an angle $\phi$ with the horizontal, it loses height $h=(n b-x) \sin \phi$ during its sliding motion. Thus the kinetic energy of the particle at the exit of the ramp is given by

$$
\frac{1}{2} m v_{x}^{\prime 2}=\frac{1}{2} m[e C(u, v)]^{2}+m g s(n b-x)
$$

where $\left(v_{x}^{\prime}, 0\right)$ is the velocity which with the particle exits the ramp. To obtain a dynamical system, we eliminate $u, v$, and $x$ in favor of $v_{x}$ by using equations (2.2) and (2.1). The last term corresponds to the acceleration due to the fact that the particle slides downhill over a distance $n b-x$. Since this distance must ne non-negative it follows that

$$
n \text { must be the smallest positive integer such that } n b-x \geq 0
$$

because that is the distance the particle slides (on the first step it encounters).

It is clear that the mass $m$ drops out of the equations. There are still many parameters left (especially through equation (2.1)). It comes as a pleasant surprise that many of these models are smoothly conjugate to one another. (The version below is an improvement over the corresponding results in $[16,17]$, since an additional parameter is essentially eliminated.)

Proposition 2.2 With the coordinate transformation

$$
\mathcal{E}=\frac{a v_{x}^{2}}{2 g b^{2} c} \quad ; \quad \kappa=\frac{s a}{c b}
$$

the dynamical system given by the equations (2.3) and (2.4) is conjugate to (if $n \neq 0$ )

$$
\mathcal{E}^{\prime}=f(\mathcal{E}, n) \equiv n e^{2}[C(\sqrt{\mathcal{E} / n}+\kappa,-a / b)]^{2}+n \kappa(1-\kappa-2 \sqrt{\mathcal{E} / n})
$$

and $n$ is determined by $\mathcal{E}$ in such a way that

$$
\mathcal{E} \in(n-1, n] \cdot \frac{(1-\kappa)^{2}}{4}
$$

When $\mathcal{E}=0$ set $\mathcal{E}^{\prime}=\kappa(1-\kappa)$, and $e \in[0,1], \kappa \in[0,1]$, while $\mathcal{E} \in[0, \infty)$.

Proof: This follows by calculation: Start by eliminating $u, v$, and $x$ from equations (2.3) and (2.4) and then apply the coordinate transformation (and use the homogeneity of $C$ ). The second part - the equation for $n$ - is easily seen to be equivalent to demanding that $n$ must be the smallest integer such that $1-\kappa-2 \sqrt{\mathcal{E} / n} \geq$ 0 . 


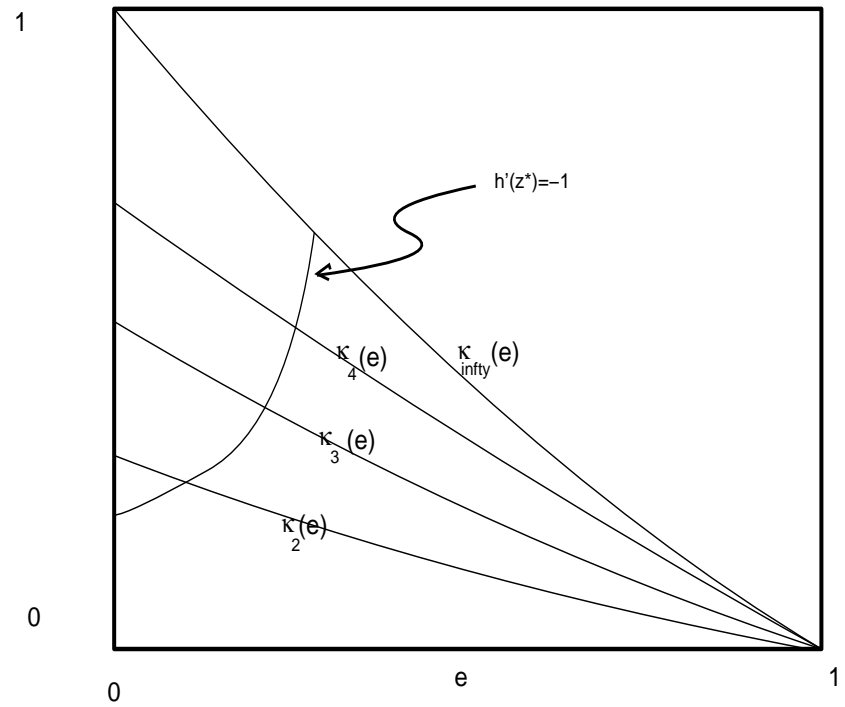

Figure 3.1: The curves $\kappa_{2}(e), \kappa_{3}(e), \kappa_{4}(e)$, and $\kappa_{\infty}(e)$ in the $e, \kappa$ plane (schematically). In the connected upper right region, the criterion map yields $I I I$. Below $\kappa_{\infty}(e)$ the velocity of any orbit is bounded from above. In addition the curve where $h^{\prime}\left(z^{*}\right)=-1$ is drawn.

\section{Main Results and Proofs}

We analyze the dynamics of the map given in proposition 2.2. Some of these results are new, in others we have supplied complete proofs.

Theorem 3.1 Given $e \in[0,1]$ and $\kappa \in[0,1]$.

i) Stopping orbits, ie $\sigma(\mathcal{E}, e, \kappa)=I$, are possible when $\kappa=0$.

ii) For $\kappa>0$, the criterion map $\sigma(\mathcal{E}, e, \kappa)$ always yields $I I$ or $I I I$.

iii) There is a function $\kappa_{\infty}(e)$ such that if $\kappa>\kappa_{\infty}(e)$, then $\sigma(\mathcal{E}, e, \kappa)=I I I$, and if $0<\kappa<\kappa_{\infty}(e)$, then $\sigma(\mathcal{E}, e, \kappa) \neq I I I$.

Remark: Statements i and ii are new. The most important statement iii is identical to that in [17], but we give a complete proof here. In fact we actually prove a little more, namely that if $\sigma(\mathcal{E}, e, \kappa)=I I I$, there is an $r>0$ such that $\mathcal{E}^{\prime} \geq \mathcal{E}+n r$. and since $n$ and $\mathcal{E}$ are related (see Proposition 2.2), this implies that $\mathcal{E}$ grows exponentially in the number of iterates. This does not mean that the particles' speed increases exponentially with time (time between jumps also grows), but it does imply that the average speed of the particle increases. Similarly, if $\sigma(\mathcal{E}, e, \kappa)=I I$ one can, from the calculations in this proof derive an upper bound on the velocity. These aspects are not found in the more schematic previous treatments.

We have a fixed point if there is an $\mathcal{E}$ such that $\mathcal{E}=f(\mathcal{E}, n)$. This equation may have solutions for every positive integer $n$. As the following result shows, all these possibilities do indeed occur.

Theorem 3.2 There is an increasing (as a function of $n$ ) family of decreasing (as a function of $e \in[0,1]$ ) curves $\left\{\kappa_{n}(e)\right\}_{n \in I N}$ with the following two properties: The family is pointwise convergent to $\kappa_{\infty}(e)$, and the system in Proposition 2.2 has a fixed point of jump number $n$ if and only if $\kappa \in\left(\kappa_{n}(e), \kappa_{\infty}(e)\right]$. (This fixed point, if it exists, is unique.)

Remark: This a more precise formulation of a similar statement in [17] which says merely that $\left\{\kappa_{n}(e)\right\}_{n \in \mathbb{N}}$ is increasing as function of $n$. The reasoning given there is essentially heuristic.

Notice that if $\kappa=\kappa_{\infty}(e)$, infinitely many fixed points do coexist. 
Theorem 3.3 i.) For given e and $\kappa$, the derivative of $f(\mathcal{E}, n)$ at the fixed point is independent of $n$.

ii.) If $\kappa>\frac{1}{4}$ and $e$ is small enough then all fixed points are unstable.

iii.) If the function $C$ and the parameter e are chosen such that $e^{2} C(x,-a / b) C^{\prime}(x,-a / b) \approx x$ at the fixed point, then all fixed points are stable.

Remark: We suspect that the region in $P$ where the fixed points are unstable is not necessarily connected.

The above result is somewhat unexpected in the sense that when $e$ is very small the particle is very nearly stopped every time it lands on the staircase. One might expect this to have a stabilizing effect! However, the opposite is the case.

In the remainder of this section we will prove the above results. Before we can do so we need some auxiliary results. The following lemma is obvious.

Lemma 3.4 Let $z=\sqrt{\mathcal{E} / n}$. Then the dynamics of Proposition 2.2 reduces to

$$
\begin{gathered}
z^{\prime 2}=\frac{n}{n^{\prime}}[h(z)]^{2} \equiv \quad \frac{n}{n^{\prime}}\left[e^{2}\left\{C\left(z+\kappa,-\frac{a}{b}\right)\right\}^{2}+\kappa(1-\kappa-2 z)\right] \\
\text { where } n \text { such that } \quad z \in\left(\sqrt{1-\frac{1}{n}}, 1\right] \cdot \frac{1-\kappa}{2} .
\end{gathered}
$$

Define the following differentiable functions:

$$
\begin{aligned}
\psi_{e}(z, \kappa) & \equiv e^{2}\left\{C\left(z+\kappa,-\frac{a}{b}\right)\right\}^{2}-(z+\kappa)^{2}+\kappa \\
\phi_{\gamma}(e, \kappa) & \equiv e^{2}\left\{C\left(\gamma \frac{1-\kappa}{2}+\kappa,-\frac{a}{b}\right)\right\}^{2}-\left(\gamma \frac{1-\kappa}{2}+\kappa\right)^{2}+\kappa \\
\text { and } \phi_{1}(e, \kappa) & \equiv \lim _{n \rightarrow \infty} \phi_{\gamma_{n}}(e, \kappa)=e^{2}\left[C\left(\frac{1+\kappa}{2},-\frac{a}{b}\right)\right]^{2}-\left[\frac{1+\kappa}{2}\right]^{2}+\kappa
\end{aligned}
$$

Here we have used the notation: $\gamma_{n} \equiv \sqrt{1-\frac{1}{n}}$.

Lemma 3.5 Fix e and $\kappa$ in $[0,1]^{2}-\{(0,0)\}$. Then

i) $z^{2}=h(z)^{2}$ has a unique solution $z^{*}(e, \kappa)$, and

ii) $z^{*}(e, \kappa)$ is differentiable with respect to $\kappa$ and $\partial_{\kappa} z^{*}(e, \kappa) \geq-\kappa /(1+\kappa)$.

Proof: i): When $\kappa=0$ we have $z^{*}=0$ is the unique fixed point. Now we have to prove that $\psi_{e}(z, \kappa)=0$ has a unique solution for all $\kappa \in(0,1]$. From Lemma 2.1 we see that $\psi_{e}(0, \kappa)>0$ and $\lim _{z \rightarrow \infty} \psi_{e}(z, \kappa)<0$. By the Intermediate Value Theorem there is a solution. To see that the solution is unique, it is sufficient (by the Mean Value Theorem) to show that $\psi$ is monotonic:

$$
e \in(0,1) \quad \Rightarrow \quad \partial_{z} \psi(z, \kappa)=2\left(e^{2} C \cdot C^{\prime}-(z+\kappa)\right) \leq 0
$$

(To avoid long formulas, we have omitted the arguments in the function $C$ as we shall do frequently from hereon. Derivatives of $C$ are always with respect to the first argument.) The cases $e=0$ and $e=1$ are easy. ii): Fixing $e$ we now use the Implicit Function Theorem:

$$
\partial_{\kappa} \psi\left(z^{*}(e, \kappa), \kappa\right)=2\left(e^{2} C C^{\prime}-\left(z^{*}+\kappa\right)\right)\left(\partial_{\kappa} z^{*}+1\right)+1=0 .
$$

From this it can easily be seen that $\partial_{\kappa} z^{*}+1>0$ (using the above equation). But also:

$$
\frac{1}{2}+\partial_{\kappa} z^{*}=-\frac{1}{2}+\left(1+\partial_{\kappa} z^{*}\right)=\frac{1-\left(z^{*}+\kappa\right)+e^{2} C C^{\prime}}{2\left(z^{*}+\kappa\right)-2 e^{2} C C^{\prime}}
$$


Since by Lemma 3.4 the fixed points must satisfy $z^{*} \leq \frac{1-\kappa}{2}$, we obtain:

$$
\begin{aligned}
\frac{1}{2}+\partial_{\kappa} z^{*} & \geq \frac{1-\frac{1+\kappa}{2}+e^{2} C C^{\prime}}{2\left(\frac{1-\kappa}{2}+\kappa\right)-2 e^{2} C C^{\prime}} \\
& =\frac{\frac{1-\kappa}{2}+e^{2} C C^{\prime}}{2\left(\frac{1+\kappa}{2}\right)-2 e^{2} C C^{\prime}}>\frac{(1-\kappa) / 2}{2((1-\kappa) / 2+\kappa)}>0
\end{aligned}
$$

From part i) of this lemma and Lemma 3.4 we conclude that there is a fixed point of jump number $n$ if and only if $z^{*}(e, \kappa) \in\left(\gamma_{n}, 1\right] \frac{1-\kappa}{2}$, where $\gamma_{n}$ is as defined above.

Lemma $3.6 i)$ : Let $e$ be in $[0,1]$. There is a unique differentiable decreasing function $\kappa_{\infty}(e)$ such that for each $e, z=\frac{1-\kappa_{\infty}(e)}{2}$ is the unique solution of $z^{2}=[h(z)]^{2}$, with $\kappa=\kappa_{\infty}(e)$.

ii): Let $e$ be in $[0,1]$. For each $n \in I N$ there is a unique differentiable decreasing function $\kappa_{n}(e)$ such that for each e, $z=\gamma_{n} \frac{1-\kappa_{n}(e)}{2}$ is the unique solution of $z^{2}=[h(z)]^{2}$, with $\kappa=\kappa_{n}(e)$.

Proof: i): This is equivalent to finding a unique root $\kappa_{\infty}(e)$ of $\phi_{1}(e, \kappa)=0$. The reasoning is the same as before; one simply checks that if $e \in(0,1)$, then

$$
\phi_{1}(e, 0)<0 \text { and } \phi_{1}(e, 1)>0 \text { and } \partial_{\kappa} \phi_{1}=e^{2} C C^{\prime}-\frac{1+\kappa}{2}+1>0 \text {. }
$$

The differentiability of $\kappa_{\infty}(e)$ follows from the Implicit Function Theorem. The cases $e=0$ and $e=1$ are easy. The fact that $\kappa_{\infty}(e)$ is a decreasing function follows easily by differentiating $\phi_{1}\left(e, \kappa_{\infty}(e)\right)$ with respect to $e$.

ii): We only do the case $e \in(0,1)$. We now have to solve $\phi_{\gamma_{n}}(e, \kappa)=0$. Existence of the solution follows as in item i). For the uniqueness, observe that $\phi_{\gamma_{n}}(e, \kappa)=0$ implies that

$$
f_{n}(\kappa) \equiv-\left(\gamma_{n} \frac{1-\kappa}{2}+\kappa\right)^{2}+\kappa \leq 0
$$

Now $f_{n}$ is quadratic in $\kappa$ and has roots $\kappa=\frac{\gamma_{n}^{2}}{\left(2-\gamma_{n}\right)^{2}}<1$ and $\kappa=1$. Since $\kappa \leq 1$, equation 3.1 implies that $\kappa$ is less than or equal to the smallest root of $f_{n}$. This in turn implies that there is an $r_{n}>0$ such that

$$
\partial_{\kappa} f_{n} \geq r_{n}>0
$$

To prove the uniqueness of the solution, note that

$$
\left.\partial_{\kappa} \phi\right|_{\gamma_{n}, e, \kappa}=e^{2} C C^{\prime} \cdot\left(1-\gamma_{n} / 2\right)+\partial_{\kappa} f_{n} \geq r_{n}
$$

It also follows from this that $\kappa_{n}(e)$ is differentiable (by the Implicit Function Theorem). The fact that $\kappa_{n}(e)$ is decreasing follows as before.

Remark: One can easily calculate that, in fact, $r_{n}=1-\gamma_{n}$. In the next proof we need that $\kappa_{n}(0)=$ $\gamma_{n}^{2} /\left(2-\gamma_{n}\right)^{2}$, which can be easily deduced from this proof, in particular by noting that it follows from $f_{n}(\kappa)=0$ (see equation 3.1). For a schematic picture of these results, see figure 3.1.

Remark: The second statement of this lemma is new.

We are now in a position to prove our main results. 
Proof of Theorem 3.2: From Lemma 3.6 we have the unique solutions $\kappa_{n}(e)$ and $\kappa_{\infty}(e)$ which are decreasing functions of $e$. By applying the Mean value Theorem twice, we obtain that there are $\tilde{\kappa}$ between $\kappa_{n}(e)$ and $\kappa_{\infty}(e)$ and $\tilde{\gamma} \in\left(\gamma_{n}, 1\right)$ such that:

$$
\begin{array}{r}
0=\phi_{1}\left(e, \kappa_{\infty}(e)\right)-\phi_{\gamma_{n}}\left(e, \kappa_{n}(e)\right)= \\
\phi_{1}\left(e, \kappa_{\infty}(e)\right)-\phi_{1}\left(e, \kappa_{n}(e)\right)+\phi_{1}\left(e, \kappa_{n}(e)\right)-\phi_{\gamma_{n}}\left(e, \kappa_{n}(e)\right)= \\
\left.\partial_{\kappa} \phi\right|_{\kappa=\tilde{\kappa}} \cdot\left(\kappa_{\infty}(e)-\kappa_{n}(e)\right)+\left.\partial_{\gamma} \phi\right|_{\gamma=\tilde{\gamma}} \cdot\left(1-\gamma_{n}\right)
\end{array}
$$

Thus $\kappa_{\infty}-\kappa_{n}>0$. We now have to prove that $\kappa_{n+1}(e) \geq \kappa_{n}(e)$. This is equivalent with $\left(\kappa_{n+1}(e), \kappa_{\infty}(e)\right] \subseteq$ $\left(\kappa_{n}(e), \kappa_{\infty}(e)\right]$ This would be implied by the statement that the existence of a fixed point with jump number $n+1$ implies the existence of a fixed point of jump number $n$. But that follows from Lemma 3.5 together with the domains given in Lemma 3.4 for orbits with jump number $n$.

Thus $\left\{\kappa_{n}(e)\right\}_{n}$ is a bounded and increasing sequence and we can set: $\lim _{n} \kappa_{n}(e)=\bar{\kappa}(e)$. From the continuity of $\phi_{\gamma}(e, \kappa)$ we obtain:

$$
\lim _{n \rightarrow \infty} \phi_{\gamma_{n}}\left(e, \kappa_{n}(e)\right)=\phi_{1}(e, \bar{\kappa}(e))=0
$$

By Lemma 3.6 part i), the last equality has a unique solution $\kappa_{\infty}(e)$ and thus

$$
\lim _{n} \kappa_{n}(e)=\bar{\kappa}(e)=\kappa_{\infty}(e)
$$

To prove the second property, first let $e=0$. From the fixed point equation $\psi_{0}(z, \kappa)=0$, we obtain $z^{*}=\sqrt{\kappa}-\kappa$. One can solve for $z^{*}$ to obtain that $z^{*} \in\left(\gamma_{n}, 1\right] \frac{1-\kappa}{2}$ iff $\kappa \in\left(\frac{\gamma_{n}^{2}}{\left(2-\gamma_{n}\right)^{2}}, 1\right]$. (See the above remark)

For $e>0$, we first prove that

$$
\kappa>\kappa_{n}(e) \Leftrightarrow z^{*}(e, \kappa)>\gamma_{n}\left(\frac{1-\kappa}{2}\right)
$$

Note that by Lemma 3.5:

$$
\left.\partial_{\kappa}\left[\gamma_{n} \frac{1-\kappa}{2}-z^{*}(e, \kappa)\right]\right|_{\kappa=\kappa_{n}(e)} \leq\left\{\frac{-\gamma_{n}}{2}-\frac{-\kappa_{n}(e)}{1+\kappa_{n}(e)}\right\} .
$$

Since $\kappa_{n}(e)$ is a decreasing function and $\kappa_{n}(0)=\frac{\gamma_{n}^{2}}{\left(2-\gamma_{n}\right)^{2}}$, we get

$$
0<\kappa_{n}(e)<\frac{\gamma_{n}^{2}}{\left(2-\gamma_{n}\right)^{2}} \Rightarrow \frac{\kappa_{n}(e)}{1+\kappa_{n}(e)} \leq \frac{\gamma_{n}^{2} /\left(2-\gamma_{n}\right)^{2}}{1+\left(\gamma_{n}^{2} /\left(2-\gamma_{n}\right)^{2}\right)}
$$

And the above expression gives $\partial_{\kappa}[\cdot]<0$. Hence, with Lemma 3.6 ii), the inequality follows.

In a similar fashion, we can prove that

$$
\kappa<\kappa_{\infty}(e) \Leftrightarrow z^{*}(e, \kappa)<\frac{1-\kappa}{2} .
$$

Proof of Theorem 3.1: i): Substitute $\mathcal{E}=0$ in Proposition 2.2.

ii): From the conditions on $C$ and Proposition 2.2 and the fact that

$$
1-\kappa-2 \sqrt{\mathcal{E} / n} \geq 0
$$


we obtain that $\mathcal{E}^{\prime}>0$. Thus $n^{\prime} \geq 1$. Continue by induction.

iii): From Lemma 3.4, we need only to consider the restriction of $z \rightarrow h(z)$ to the interval $\left(\gamma_{n}, 1\right] \frac{1-\kappa}{2}$. Noting that $h(0)>0$ and $h$ is continuous, we now have two cases:

$$
\begin{aligned}
& \kappa>\kappa_{\infty}(e) \Rightarrow h(z)^{2}-z^{2}>0 \text { for all } z \in(0,1] \frac{1-\kappa}{2} . \\
& \kappa<\kappa_{\infty}(e) \Rightarrow h(z)^{2}-z^{2}=0 \text { has a solution } z \in(0,1] \frac{1-\kappa}{2} .
\end{aligned}
$$

In the first case we obtain (by compactness) that there is an $r>0$ such that (from Lemma 3.4)

$$
z \in\left[0, \frac{1-\kappa}{2}\right] \Rightarrow h(z)^{2}>z^{2}+r \Rightarrow n^{\prime} z^{\prime 2} \geq n z^{2}+n r \Rightarrow \mathcal{E}^{\prime} \geq \mathcal{E}+n r \quad .
$$

Because $n$ is related to $\mathcal{E}$ by $n-1<4 \mathcal{E} /(1-\kappa)^{2} \leq n$ exponential growth follows.

In the second case, we see from Theorem 3.2 that the graph of $h(z)$ meets (see Figure 4.1) the diagonal exactly once, namely at some $z^{*}(e, \kappa)<\frac{1-\kappa}{2}$. Using the convention that $\gamma_{n}=\sqrt{1-\frac{1}{n}}$, we see that there must be an natural number $n_{0}>0$ such that $z^{*}(e, \kappa)<\gamma_{n_{0}}\left(\frac{1-\kappa}{2}\right)$. And thus, if $n \geq n_{0}$, there is $r_{1}(n)>0$ so that for all $z \in\left(\gamma_{n}, 1\right] \frac{1-\kappa}{2}$

$$
h(z)^{2}-z^{2}<-r_{1},
$$

If $n \leq n_{0}$, then there are $r_{2}(n)>0$ and $r_{3}(n)>0$ such that for all $z \in\left(\gamma_{n}, 1\right] \frac{1-\kappa}{2}$

$$
r_{2} \geq h(z)^{2}-z^{2} \geq-r_{3}
$$

Thus for jump numbers smaller than to $n_{0}$ (that is: $\left.\mathcal{E}<\left(n_{0}-1\right)\left(\frac{1-\kappa}{2}\right)^{2}\right)$ we have

$$
\mathcal{E}-n r_{3} \leq \mathcal{E}^{\prime} \leq \mathcal{E}+n_{0} r_{2}
$$

And for jump numbers greater than or equal to $n_{0}$ :

$$
\mathcal{E}^{\prime} \leq \mathcal{E}-n r_{3}
$$

From this, one easily sees that $\lim _{\sup _{i}} \mathcal{E}_{i} \leq n_{0}\left(\left(\frac{1-\kappa}{2}\right)^{2}+r_{2}\right)$.

Proof Theorem 3.3: i): We will consider the derivative of $f(\mathcal{E}, n)$ at its fixed point. Since $\mathcal{E} \rightarrow z(\mathcal{E})$ is a diffeomorphism (for fixed $n$ ), this is the same as the derivative of $h(z)$ at its fixed point. The latter is independent of the jump number and so the stability of the fixed point is also independent of the jump number $n$.

ii): From Lemma 3.4, we see that when $z^{*}=h\left(z^{*}\right)$

$$
\frac{d z^{*}}{d z}=h^{\prime}\left(z^{*}\right)=\frac{e^{2} C\left(z^{*}+\kappa,-a / b\right) C^{\prime}\left(z^{*}+\kappa,-a / b\right)-\kappa}{z^{*}} .
$$

Using the conditions on $C$ it is easy to see that

$$
h^{\prime}\left(z^{*}\right) \leq 1
$$

So the criterion for the stability of the fixed point is:

$$
\begin{aligned}
& e^{2} C C^{\prime}+z^{*}-\kappa>0 \quad \Rightarrow \quad h^{\prime}\left(z^{*}\right)>-1 \Rightarrow \text { stability } . \\
& e^{2} C C^{\prime}+z^{*}-\kappa<0 \quad \Rightarrow \quad h^{\prime}\left(z^{*}\right)<-1 \Rightarrow \text { instability . }
\end{aligned}
$$


Now, when $e=0$, the solutions of $\psi_{0}(z, \kappa)=0$ are easy to find. The fixed point is given by $z^{*}=\sqrt{\kappa}-\kappa$ and

$$
h^{\prime}\left(z^{*}\right)=\leq-1 \text { if } \kappa \geq \frac{1}{4}
$$

Since $\kappa_{\infty}(0)=1$, this proves ii). Item iii) is straightforward.

Remark: An extra condition on $C$ " would give us a curve $e_{s}(\kappa)$ as the locus of $h^{\prime}\left(z^{*}\right)=-1$ (see Figure 3.1). One could insure that $h^{\prime}\left(z^{*}(e, \kappa)\right)=-1$ has a unique solution. This can be done using the same strategy as before. In Figure 3.1 we have drawn this schematically (see also [16]).

Remark: Since we know that for $\kappa>\kappa_{\infty}(e)$, the behavior the energy of the particle must be eventually increasing, it is easy to conclude that this means that in Proposition $2.2, \mathcal{E}=\infty$ is an attractor. One shows that if we set $n=\frac{4 \mathcal{E}}{(1-\kappa)^{2}}+\mathcal{O}\left(\mathcal{E}^{-1}\right)$, the equation in Proposition 2.2 linearizes to

$$
\mathcal{E}^{\prime}=\mathcal{E} \frac{4 e^{2}}{(1-\kappa)^{2}} C^{2}\left(\frac{1+\kappa}{2}, \frac{-a}{b}\right)+\mathcal{O}(1)
$$

Thus $\kappa_{\infty}(e)$ is determined by setting the coefficient of $\mathcal{E}^{\prime}$ equal to one in this equation.

\section{Unstable Behavior and Periodic Orbits}

In this section is dedicated to a more detailed analysis of orbits in the $\sigma=I I$ region. The first group of results concerns the existence of unstable behavior in the upper left corner of the $I I$ region (see Figure 3.1). The second concerns the related issue of the existence of periodic orbits with non-constant jump number. The results in this section are considerably more detailed than the corresponding results in $[16,17]$. In fact, one of the main results (theorem 4.5) is new.

Given an orbit we will consider the sequence of jump numbers $\left\{n_{i}\right\}_{i=1}^{\infty}$. Define

$$
\delta n \equiv \frac{\frac{1}{2}\left(\lim \sup n_{i}-\liminf n_{i}\right)}{\frac{1}{2}\left(\lim \sup n_{i}+\liminf n_{i}\right)}
$$

We assume $h$ is twice differentiable (although continuously differentiable is enough).

In the following suppose we have a fixed point $z^{*}$ of $z \rightarrow h(z)$ and for given $e$ define:

$$
\begin{gathered}
\lambda \equiv\left|h^{\prime}\left(z^{*}\right)\right| . \\
\lambda_{\infty} \equiv \lim _{\kappa / \kappa_{\infty}(e)}\left|h^{\prime}\left(z^{*}\right)\right| .
\end{gathered}
$$

Recall that in the $I I$-region $\lambda>1$ and if $e$ is close to 0 , then the solution of

$$
\phi_{1}\left(e, \kappa_{\infty}(e)\right)=0
$$

yields that $\kappa_{\infty}(e)$ is close to 1 (see also equation (3.2)). Thus the fixed points in this case satisfy

$$
h^{\prime}\left(z^{*}\right) \approx \frac{-\kappa}{z^{*}} \approx \frac{-\kappa}{\frac{1}{2}(1-\kappa)},
$$

which may have an arbitrarily large modulus.

Theorem 4.1 For fixed e small enough (so that $\lambda_{\infty}>1$ )

$$
\lim _{\kappa / \kappa_{\infty}(e)} \delta n \leq \frac{\lambda_{\infty}-1}{\lambda_{\infty}+1} \leq 1
$$


Proof: Since by assumption $\lambda_{\infty}>1$ the second inequality is obvious. Following [17], we define (see Figure 4.1)

$$
\begin{aligned}
& z_{-} \quad h\left(\frac{1-\kappa}{2}\right) \\
& z_{+} \text {such that } h\left(z_{+}\right) \quad=\quad \frac{1-\kappa}{2} \\
& n_{-} \text {largest integer such that } z_{-}>\sqrt{1-\frac{1}{n_{-}}} \frac{1-\kappa}{2} \\
& n_{+} \text {smallest integer such that } z_{+}<\sqrt{1-\frac{1}{n_{+}}} \frac{1-\kappa}{2}
\end{aligned}
$$

For $e$ sufficiently close to 0 and $\kappa$ sufficiently close to $\kappa_{\infty}(e)$ we may assume that $h$ restricted to the interval $\left[\sqrt{1-\frac{1}{n_{-}}}, 1\right] \cdot \frac{1-\kappa}{2} \approx\left[z_{-}, \frac{1-\kappa}{2}\right]$ is very close to being affine. We will approximate $h$ with the affine function

$$
h(z)=\lambda\left(z_{+}-z\right)+\frac{1-\kappa}{2} .
$$

Since (see Figure 4.1) $\left[z_{+}, \frac{1-\kappa}{2}\right]$ is mapped to $\left[z_{-}, \frac{1-\kappa}{2}\right]$, we obtain:

$$
\lambda=\frac{\frac{1-\kappa}{2}-z_{-}}{\frac{1-\kappa}{2}-z_{+}}=\frac{n_{+}}{n_{-}}+n_{+} \mathcal{O}\left(\frac{1}{n_{-}^{2}}\right) .
$$

Here we have used that

$$
z=\sqrt{1-\frac{1}{n}} \frac{1-\kappa}{2}+\mathcal{O}\left(\frac{1}{n^{2}}\right),
$$

and similarly for $n_{+}$and $n_{-}$(equations 4.4). Thus we find that

$$
\frac{\lambda-1}{\lambda+1}=\frac{\frac{1}{2}\left(n_{+}-n_{-}\right)}{\frac{1}{2}\left(n_{+}+n_{-}\right)}+\mathcal{O}\left(\frac{1}{n_{-}}\right)
$$

The proof of the theorem is now implied by the first parts of the following two lemmas:

Lemma 4.2 Suppose that the jump number decreases, so that $n^{\prime}<n$. Then

i): $n_{-} \leq n$.

ii): $\left.n^{\prime} \geq \operatorname{ceil}\left(n-\frac{n}{n_{-}}\right)\right)$.

Proof: i): From the definition of $z_{-}$and the domain of $z$ (Lemma 3.4) we know that $z_{-}<h(z)$. If $n^{\prime}<n$ (see also Figure 4.1) we conclude that in addition $h(z) \leq \sqrt{1-\frac{1}{n}} \cdot \frac{1-\kappa}{2}$. Thus $n_{-} \leq n$ follows from the definition of $n_{-}$.

ii): Since clearly $z \leq \frac{1-\kappa}{2}$, we have

$$
z^{\prime}=\sqrt{\frac{n}{n^{\prime}}} h(z) \geq \sqrt{\frac{n}{n^{\prime}}} h\left(\frac{1-\kappa}{2}\right)
$$

so that

$$
\mathcal{E}^{\prime} \geq n z_{-}^{2} \geq n\left(1-\frac{1}{n_{-}}\right)\left(\frac{1-\kappa}{2}\right)^{2}
$$

Recalling

$$
n^{\prime}=\operatorname{ceil}\left(\frac{4 \mathcal{E}^{\prime}}{(1-\kappa)^{2}}\right)
$$

we obtain the second statement. 


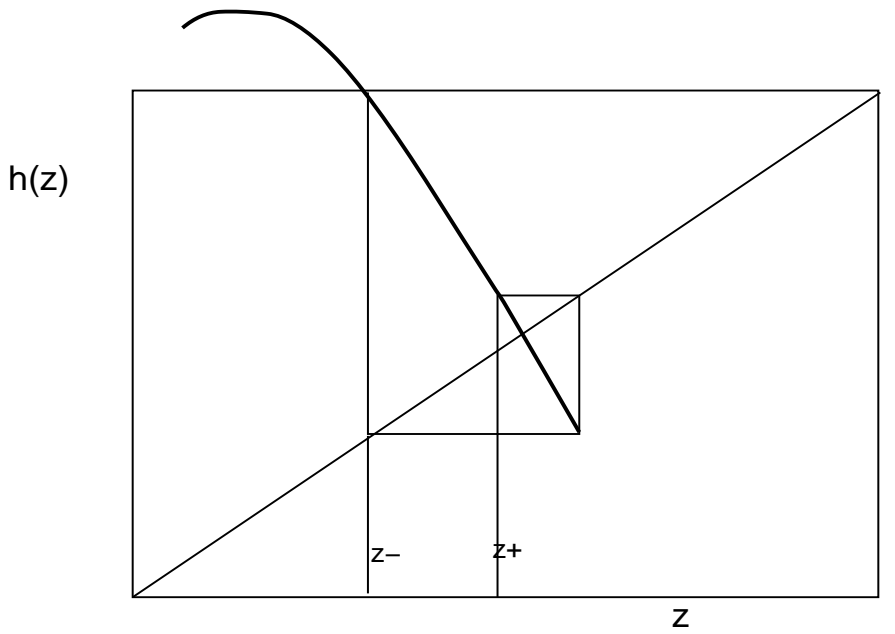

Figure 4.1: The function $h(z)$ together with $z_{+}$and $z_{-}$.

Lemma 4.3 Suppose that the jump number increases, so that $n^{\prime}>n$. Then

i): $n \leq n_{+}$.

ii): $n^{\prime} \leq \operatorname{ceil}\left(n+\lambda\left(1-\frac{n}{n_{+}}\right)+\frac{\lambda^{2}}{4 n}\left(1-\frac{n}{n_{+}}\right)^{2}\right)$.

Proof: i): Analogously, if $n^{\prime}>n$, then we must have from Lemma 3.4 (or Figure 4.1) that $h(z)>h\left(z_{+}\right)=$ $\frac{1-\kappa}{2}$. Thus $z<z_{+}$, and $n \leq n_{+}$follows from the definition of $n_{+}$.

ii): We observe that $z^{\prime} \leq \sqrt{\frac{n}{n^{\prime}}} h\left(\sqrt{1-\frac{1}{n}} \frac{1-\kappa}{2}\right)$. Use equation 4.5 to obtain that

$$
\mathcal{E}^{\prime}<n\left(1+\frac{\lambda}{2}\left(\frac{1}{n}-\frac{1}{n_{+}}\right)\right)^{2}\left(\frac{1-\kappa}{2}\right)^{2}
$$

Using the same relation for $n^{\prime}$ as in the previous lemma,

$$
n^{\prime} \leq \operatorname{ceil}\left(n\left(1+\frac{\lambda}{2}\left(\frac{1}{n}-\frac{1}{n_{+}}\right)\right)^{2}\right) .
$$

From this the second statement follows.

This finishes the proof of Theorem 4.1.

Remark: Note that the lemmas actually yield statements about the dynamics of the system. Not only do they tell us that if the jump number is not constant, it eventually varies between $n_{-}$and $n_{+}$, but that it changes only by small increments. (These lemmas were not included in earlier versions).

Now we turn to the existence of non-trivial periodic orbits (that is: with non-constant jump number). The previous result suggests that if there are periodic orbits then $n_{-} \leq n \leq n_{+}$. Thus if $n_{-} \geq n_{+}$we expect that non-trivial periodic orbits do not exist. We prove this without requiring that $h$ is close to linear. In fact, the existence of non-trivial periodic orbits in this system is closely related to unstable behavior. In the following we use the definition of $z_{-}, z_{+}, n_{-}$and $n_{+}$exactly as in the proof of Theorem 4.1.

Proposition 4.4 If $h$ is such that either i) $h^{\prime}(z) \in[0,1]$ or ii) $h^{\prime}(z) \in[-1,0]$ and $n_{+}<n_{-}$, then there are no non-trivial periodic orbits. 
Proof: First assume that $h$ is increasing. One sees by comparing Proposition 2.2 and Lemma 3.4 that then $f$ is monotone increasing as well. Thus in this case there can be no periodic orbit $(f$ preserves the order on the line).

Now assume that $h$ is non-increasing. Note that $z_{+}<z_{-}$. For a non-trivial periodic orbit to exist we must have that at a certain point $P$ along this orbit $n^{\prime}>n$. One first proceeds as in the above lemmas to show that in this case:

$$
\begin{aligned}
& n^{\prime}>n \quad \Rightarrow \quad n \leq n_{+} \\
& n^{\prime} \geq n \quad n \leq n_{+}
\end{aligned}
$$

Next observe that from the definition of $z_{+}$:

$$
\begin{aligned}
h(z) & =h\left(z_{+}\right)+\left(h(z)-h\left(z_{+}\right)\right) \\
\leq h\left(z_{+}\right)+\left(z_{+}-z\right) & =\left(1+\sqrt{1-\frac{1}{n_{+}}}-\sqrt{1-\frac{1}{n}}\right) \frac{1-\kappa}{2}<\left(1+\frac{1}{n}\right) \frac{1-\kappa}{2}
\end{aligned}
$$

From this and the previous paragraph we conclude that:

$$
n \leq n_{+} \Rightarrow n^{\prime}=n \text { or } n^{\prime}=n+1
$$

On the other hand it is easy to see that

$$
n_{+}<n<n_{-} \Rightarrow n^{\prime}=n
$$

This relation and the previous imply that starting at the point $P$ the sequence of jump numbers is nondecreasing and bounded, and therefore eventually constant.

Remarks: With a little effort this result can be slightly generalized in two ways. The condition that $h^{\prime}(z) \in[0,-1]$ implies that $n_{+} \leq n_{-}+1$. The second condition in the Proposition, namely that $n_{+}<n_{-}$ can be dropped. Furthermore, if there is an $N$ such that condition on the Proposition holds for $z \in I_{N}=$ $\left(\sqrt{1-\frac{1}{N}}, 1\right] \frac{1-\kappa}{2}$, then there are no periodic orbits with all jump numbers greater than $N$.

However, in the unstable regime there are plenty of periodic orbits as the following result shows.

Theorem 4.5 For any $N \in \mathbb{N}$ there is an e sufficiently small and $\kappa$ close to $\kappa_{\infty}(e)$ (but smaller) such that the dynamical system given by Proposition 2.2 contains a full shift on $N$ symbols.

Sketch of Proof: From Proposition 2.2 one sees that $f$ has infinitely many branches $\left.f_{n} \equiv f\right|_{I_{n}}$ where $I_{n}=(n-1, n] \frac{1}{4}(1-\kappa)^{2}$. The right endpoints of these branches lie on the line $y=\omega x$ where

$$
\omega=e^{2}\left[C\left(\frac{1+\kappa}{2}, \frac{-a}{b}\right)\right]^{2} \frac{4}{(1-\kappa)^{2}},
$$

which approaches 1 from above as $\kappa \nearrow \kappa_{\infty}(e)$ (see also equation 3.2). In addition one sees from Proposition 2.2 and Lemma 3.4 that for large $n$ the slope of these branches of $f$ is very nearly constant

$$
\partial_{\mathcal{E}} f=\sqrt{\frac{n}{\mathcal{E}}}\left(e^{2} C C^{\prime}-\kappa\right) \approx 2 \frac{e^{2} C C^{\prime}-\kappa}{1-\kappa} .
$$

This becomes very large in modulus if in addition $e$ is close to 0 so that $\kappa$ and $\kappa_{\infty}(e)$ are close to 1 .

From these facts it follows easily that for $e$ small enough and $\kappa$ close enough to $\kappa_{\infty}(e)$ there is an interval $[a, b]$ such that there are at least $N$ branches $f_{i}$ of $f$ with the property that

$$
\inf _{x \in I_{i}} f_{i}(x) \leq a \text { and } \sup _{x \in I_{i}} f_{i}(x) \geq b
$$

This implies that each of these expanding branches maps a subinterval of $[a, b]$ onto $[a, b]$.

Remark: This emphasizes again that the behavior of this system is extremely unstable for very small coefficients of restitution, a fact that does not seem to be - for the author — very intuitive. 


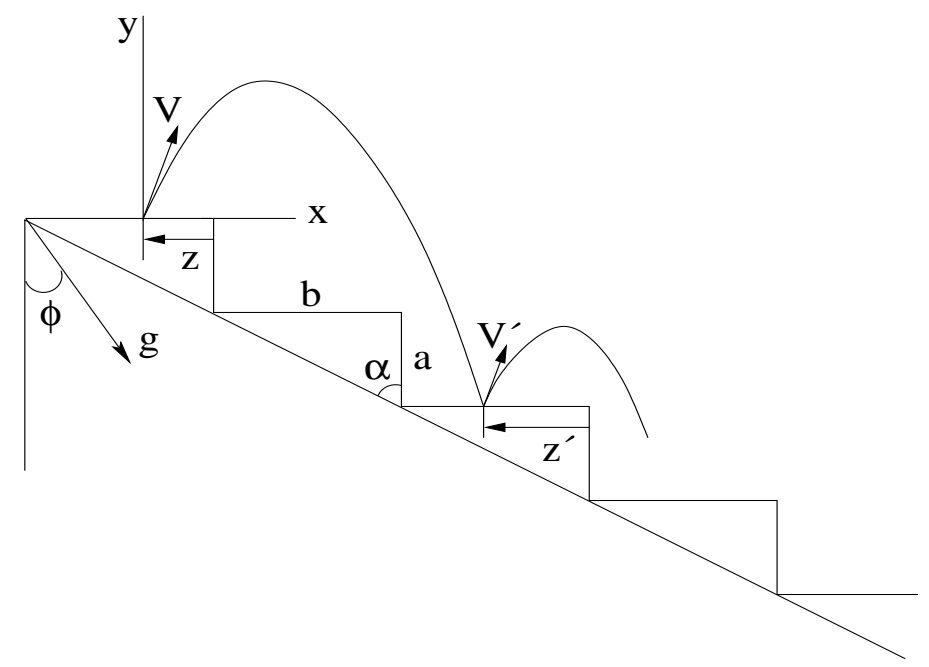

Figure 5.1: The model where bouncing is allowed.

\section{A Model Allowing Vertical Bouncing.}

We will now briefly set the previous results against those of a more complicated model where vertical bouncing is allowed (see the figure in this section). Lifting the restriction on vertical bouncing, we obtain a new class of models. We will summarize the model, and the main results, without giving the proofs (however, see [19]).

In the figure in this section we depicted a point particle of mass $m$ falling down a staircase with vertical bouncing. Again the steps of the staircase are rectangular (with sides of length $a$ and $b$ ) and the staircase itself is forward inclined over an angle $\phi$. The dynamical space $X$ is now 3-dimensional, because in order to know the particles' landing point, we must know where it took off ( $z$ in the figure) and both components of the velocity ( $v_{x}$ and $v_{y}$ at the point of take-off). Similarly the parameter space is also a little more complicated. The law of restitution is now:

$$
\begin{aligned}
& u^{\prime}=e_{t} C_{1}(u, v) \\
& v^{\prime}=e_{n} C_{2}(u, v) .
\end{aligned}
$$

Here, as before, $(u, v)$ is the velocity of the particle just before landing and $\left(u^{\prime}, v^{\prime}\right)$ just after. Using a coordinate transform similar to the one before, one can show that the only parameters one needs to formulate the dynamical system are: $e_{t}, e_{n}$, and $\kappa$ (with the same definition as before). Thus the parameter space $P$ is 3-dimensional as well. Because of the extra complication of this model, we will assume (with $[18,19])$ the simplest possible law of restitution, namely:

$$
\begin{aligned}
& u^{\prime}=e_{t} u \\
& v^{\prime}=-e_{n} v .
\end{aligned}
$$

After the coordinate change, the resulting dynamical system can now be written as: $F\left(v_{x}, v_{y}, z\right) \rightarrow$ $\left(v_{x}^{\prime}, v_{y}^{\prime}, z^{\prime}\right)$ where

$$
\begin{aligned}
v_{x}^{\prime} & =e_{t}\left[v_{x}+v_{y}+\sqrt{v_{y}^{2}+n}\right] \\
v_{y}^{\prime} & =e_{n} \sqrt{v_{y}^{2}+n} \\
z^{\prime} & =n(1-\kappa)+z-2 \kappa\left(v_{x}+v_{y}\right)\left(v_{y}+\sqrt{v_{y}^{2}+n}\right)
\end{aligned}
$$

As one can see from the figure, $n$ is determined by the requirement that

$$
n \text { smallest non negative integer such that } z^{\prime} \geq 0 \text {. }
$$


It is worth noting that the model studied by [15] can be obtained from this model by setting $e_{t}=e_{n}=0$ and taking a limit as the stepsize of the staircase tends to zero (see [19] for details).

Our main interest is to understand the criterion map $\sigma: X \times P \rightarrow\{I, I I, I I I\}$. In the previous model we were able to determine all the main features of this map. Here we have a little more trouble. In this case it has not yet been been established that $\sigma^{-1}(I I I)$ consists of initial conditions whose orbits have eventually increasing speeds (although it appears this is true).

Theorem 5.1 Let $p=\left(e_{t}, e_{n}, \kappa\right) \in[0,1]^{3}=P$ and $x=\left(v_{x}, v_{y}, z\right) \in \mathbb{R}^{2} \times[0,1]=X$. There are smooth surfaces $\kappa_{\infty}\left(e_{t}, e_{n}\right)$ and $\kappa_{s}\left(e_{t}, e_{n}\right)$ in $P$ with the following properties:

$$
0 \leq \kappa_{\infty}\left(e_{t}, e_{n}\right) \leq \kappa_{s}\left(e_{t}, e_{n}\right) \leq 1
$$

where equality is only possible in the boundary of $[0,1]^{2}$. Furthermore,

$$
\begin{aligned}
\kappa<\kappa_{\infty} & \Rightarrow \sigma(x, p) \in\{I, I I\} \\
\kappa_{\infty} \leq \kappa \leq \kappa_{s} & \Rightarrow \sigma(x, p) \in\{I, I I, I I I\} \text { \& orbits of all three types occur } \\
\kappa>\kappa_{s} & \Rightarrow \sigma(x, p) \in\{I I, I I I\}
\end{aligned}
$$

For proofs see [19]. The main open question for this class of models is the following conjecture.

Conjecture $5.2 \sigma(x, p)=I I$ only if $\kappa_{\infty} \leq \kappa \leq \kappa_{s}$.

If the above can be proved, it may help to explain the fact that when one pours sand on top of a sand pile there is an interval of angles that are stable under the pouring process (see [7]).

In principle, once a particle stops, it can be held in place by what is known static friction ([10]). However, it turns out that depending on the shape and and smoothness of the particle static friction can differ greatly: smooth and rounded particle may have a marked tendency to continue rolling or sliding which influences the dynamics of the avalanches (see [4]). In [19] we proposed a hybrid model, where the particle is allowed to bounce, and also, upon stopping on a certain step, it is allowed to slide without friction down the step to continue its motion. In this version, orbits of type $I$ are replaced, presumably by orbits of type $I I$, and the model gains some characteristics of the previous model (it may acquire unstable periodic orbits, and has no stopping orbits). On the whole though, the dynamics appears to be even more complicated than the models considered here.

\section{References}

[1] P. Bak, C. Tang, K. Wiesenfeld, Self-Organized Criticality: An Explanation of $1 / f$ Noise, Phys. Rev. Lett. 59, NO 4, 381-384, 1987.

[2] R. Cross, The Coefficient of Restitution for Happy Balls, Unhappy Balls, and Tennis Balls, Am. J. Phys. 68, No 11, 1025-1031, 2000

[3] S. F. Foerster, M. Y. Louge, H. Chang, K. Allia, Measurement of the Collision Properties of Small Spheres, Phys. Fluids 6, 1108-1115, 1994.

[4] V. Frette, K. Christensen, P. Meakin, Avalanche Dynamics in a Pile of Rice, Nature 379, 49-52, 1996.

[5] C. Henrique, M. A. Aguirre, A. Calvo, I. Ippolito, S. Dippel, G. G. Batrouni, D. Bideau, Energy Dissipation and Trapping of Particles Moving on a Rough Surface, Phys. Rev. E 57, 4743, 1998. 
[6] J. Hermans, A Symmetric Sphere Rolling on a Surface, Nonlinearity 8, No 4, 493-515, 1995.

[7] H. M. Jaeger, C.-h. Liu, S. R. Nagel, Relaxation at the Angle of Repose, Phys. Rev. Lett. 62, No1, 40-43, 1989.

[8] H. M. Jaeger, S. R. Nagel, R. P. Behringer, The Physics of Granular Materials, Physics Today, April 1996, 32-38.

[9] H. M. Jaeger, S. R. Nagel, R. P. Behringer, Granular Solids, Liquids and Gases, Rev. Mod. Phys. 68, No 4, 1259-1273, 1996.

[10] J. Lee, Avalanches in 1+1 Dynamical Piles: a Molecular Dynamics Study, J. de Physique I, 3, 20172027, 1993.

[11] P. A. Lemieux, D. J. Durian, From Avalanches to Fluid Flow: A Continuous Picture of Grain Dynamics Down a Heap, Phys. Rev. Lett. 85, No 20, 4273-4276, 2000.

[12] G. Ristow, F. X. Riguidel, D. Bideau, Different characteristics of the Motion of a Single Particle on a Bumpy Inclined Line, J. Phys. I, 4, 1161, 1994.

[13] L. Samson, I. Ippoliti, D, Bideau, G. G. Batrouni, Motions of Grains down a Bumpy Surface, Chaos Vol 9, N0 3, 639-648, 1999.

[14] W. J. Stronge, Unraveling Paradoxical Theories for Rigid Body Collisions, J. Appl. Mech. 58, 10491055, 1991.

[15] A. Valance, D. Bideau, Dynamics of a Ball on a Rough Inclined Line, Phys Rev. E 57, 1886, 1998.

[16] G. L. Vasconcelos, J. J. P. Veerman, Geometrical Model for a Particle on a Rough Inclined Surface, Phys. Rev. E 59, 5641-5646, 1999.

[17] G. L. Vasconcelos, J. J. P. Veerman, Geometrical Models for Grain Dynamics, Physica A 271, 251-259, 1999.

[18] G. L. Vasconcelos, F. V. Cunha-Jr, J. J. P. Veerman, Chaotic Behavior in a Model for grain Dynamics, Physica A, 295 (1-2), 261-267, 2001.

[19] J. J. P. Veerman, F. V. Cunha-Jr, G. L. Vasconcelos, Dynamics of a Granular Particle on a Rough Surface with a Staircase Profile, Physica D 168/169, 220-234, 2002. 